\title{
MAXIMAL IDEALS IN THE GROUP ALGEBRA OF AN EXTENSION, WITH APPLICATIONS
}

\author{
BY
}

\author{
ARNOLD J. INSEL
}

\begin{abstract}
Let $A$ be a closed central subgroup of $E$ (all groups are locally compact and second countable). Let $G=E / A$. For each $a \in \hat{A}$, the dual of $A$, a multiplication is introduced with respect to which the Banach space $L^{1}(G)$ is a Banach algebra, denoted by $L^{1}(G, a(\sigma))$. A one-to-one correspondence is established between the maximal closed (right, left, 2 -sided) ideals of the group algebra $L^{1}(E)$ and the totality of maximal closed (right, left, 2 -sided) ideals of $L^{1}(G, a(\sigma))$, where $a$ varies over $\hat{A}$. Applications include a bound for the spectral norm of an element of $L^{1}(E)$ and the representation of a continuous positive definite function on $E$ as an integral (a 'Bochner' theorem).
\end{abstract}

Introduction. G. P. Johnson [6] characterized the maximal ideal space of a generalized group algebra $B^{1}(G, \mathcal{Q})$, where $G$ is a locally compact abelian group and $\mathbb{Q}$ is a commutative Banach algebra. In particular if $\mathbb{Q}=L^{1}(A)$, the group algebra of locally compact abelian group $A, B^{1}(G,(1)$ is isomorphic to the group algebra of $A \times G$ and so one can characterize the maximal ideal space of $L^{1}(A \times G)$ in terms of those of $L^{1}(A)$ and $L^{1}(G)$, namely the product.

Our program is to consider any locally compact second countable groups $G, A$, and $E$, such that $A$ is abelian and $E$ is a central extension of $G$ by $A$, i.e. $A$ is a closed central subgroup of $E$, and $G$ is topologically isomorphic to $E / A$. Under these conditions we are interested in determining the maximal closed ideals (left, right and two-sided) of the group algebra $L^{1}(E)$. An important tool will be a "partial" Fourier transform defined on $L^{1}(E)$ and taking an element $f$ into a function $\hat{f}$ defined on $\hat{A}$, the dual of $A$, with values in various Banach algebras, a different one for each $a \in \hat{A}$. We offer two applications of our results one of which leads to a Bochner theorem involving the representation of any continuous positive definite function on $E$ as an integral over a vector valued measure on $\hat{A}$ with values in $L^{\infty}(G)$.

The author would like to express his appreciation to Professor Calvin C. Moore under whose direction this research was done as a part of the author's $\mathrm{Ph}$. D. thesis.

Presented to the Society, January 22, 1971 under the title $O_{n}$ the group algebra of an extension; received by the editors May 12, 1971.

AMS (MOS) subject classifications (1970). Primary 22D15, 43A20; Secondary 43A30, 43A35.

Key words and phrases. Topological group extension, group algebra, maximal closed ideal, spectral norm, positive definite function, Bochner theorem. 
I. For the rest of this paper we fix groups $A, E$, and $G$ as in the Introduction. As a result of the work done by G. W. Mackey [13] and [14] we have a method of constructing $E$ from $A$ and $G$ and a 2-cocycle as in the discrete theory of groups but with the additional requirement that the 2-cocycle be a Borel function. For the details see Mackey. For a more general treatment of the cohomology of topological groups in the style of Eilenberg-Mac Lane see C. C. Moore [16]. For the rest of this paper we fix a Borel 2-cocycle $\sigma: G \times G \rightarrow A$ which characterizes the way $E$ is an extension of $G$ by $A$. The product $A \times G$ becomes a group under the multiplication $(a, g)(b, b)=(a b \sigma(g, b), g b)$. With an appropriate topology this group becomes topologically isomorphic to $E$, with $A$ corresponding to the set of all ordered pairs whose second coordinate is 1 . For the details see Mackey. Henceforth we view $E$ in this manner. We note that the Borel structure for $E$ is the product of the Borel structures for $A$ and $G$ and that the Haar measure for $E$ can be taken as the product of the Haar measures for $A$ and $G$. The Fubini theorem applies. All Haar measures in this paper shall be assumed left-invariant.

Our next concern is to properly view $L^{1}(E)$ as an algebra of functions defined on $G$ with values in $L^{1}(A)$. This viewpoint has been worked out by H. Leptin [9]. We use a modification of his approach, simplified by the fact that $G$ acts trivially on $A$. To be consistent with Mackey's approach we must modify Leptin's definition of the measurable factor system $P$ obtained from 2-cocycle $\sigma$. We define $P(x, y)(b)(t)=b\left(\sigma^{-1}(x, y) t\right)$ for all $b \in L^{1}(A), x, y \in G$ and $t \in A$. Then allow $L^{1}\left(G, L^{1}(A) ; P\right)$ to denote the space of all classes of functions from $g$ with values in $L^{1}(A)$, integrable with respect to the Haar measure of $G$. For the theory of integration of vector valued functions see [2] or [3]. Then for f. $g \in L^{1}\left(G, L^{1}(A) ; P\right)$ and $x \in G$, define

$$
f * g(x)=\int_{G} P\left(x y, y^{-1}\right)\left(f(x y) * g\left(y^{-1}\right)\right) d y .
$$

Then define $\Sigma: L^{1}(E) \rightarrow L^{1}\left(G, L^{1}(A) ; P\right)$ by $\Sigma f(x)(t)=f(x, t)$. Then by [9, p. 264] $\Sigma$ is an algebra isomorphism preserving norm. We shall implicitly identify these two algebras via $\Sigma$.

We complete this section by making some observations about ideals in $L^{1}(E)$.

1.1 Definition. Let $S$ be a subset of $L^{1}(A)$. Define $F(S)$ as a subset of $L^{1}(E)=L^{1}\left(G, L^{1}(A) ; P\right)$ as follows: $F(S)=\left\{f \in L^{1}(E): f(x) \in S\right.$ for almost all $x \in G$.

1.2 Proposition. For any closed ideal $I$ in $L^{1}(A), F(I)$ is a closed twosided ideal in $L^{1}(E)$.

For a proof see $[10]$. 
1.3 Proposition. Let $K$ be a Borel ideal in $L^{1}(A)$. Then $F(\bar{K})=\overline{F(K)}$.

Proof. Since $F(\bar{K})$ is closed $\overline{F(K)} \subset F(\bar{K})$. Now suppose $f \in F(\bar{K})$. Define $G_{a}=\{x: f(x) \in K\}$ and $G_{b}=\{x: f(x) \in \bar{K} \sim K\}$. Then without loss of generality assume $G_{a} \cup G_{b}=G$. If $G_{b}$ is of measure zero there is nothing to prove. Now suppose that $0<\mu\left(G_{b}\right)<\infty$, where $\mu$ is the Haar measure on $G$. Consider any $\epsilon>0$. Cover $\bar{K} \sim K$ with a sequence $\left\{S_{n}\right\}$ of open balls in $L^{1}(A)$ each of radius less than $\epsilon / 2 \mu\left(G_{b}\right)$. For each $n$ choose an $b_{n} \in S_{n} \cap K$. Now define $f_{\epsilon} \in L^{1}(E)$ as follows: $f_{\epsilon}=f$ on $G_{a}$. For $x \in G_{b}$ let $f_{\epsilon}(x)=b_{n}$, where $n$ is the smallest integer for which $f(x) \in S_{n}$. Then clearly $f_{\epsilon}$ is a Borel function from $G$ into $L^{1}(A)$, and furthermore $f_{\epsilon}(G) \subset K$. Also $\int_{G}\|f(x)\| d x<\infty$ and in fact it is easily seen that

$$
\int_{G}\|f(x)-f(x)\| d x=\left\|f_{\epsilon}-f\right\|_{1} \leq \epsilon
$$

Hence $f \in \overline{F(K)}$.

Now suppose that $\mu\left(G_{b}\right)=\infty$. Then $G_{b}$ is a union of an increasing sequence of Borel subsets $\left\{C_{b}^{n}\right\}$, each of finite measure. Now define $G^{n}=G_{a} \cup G_{b}^{n}$. Then define $f_{n} \in L^{1}(E)$ to be $f$ on $G^{n}$ and zero off $G^{n}$. Then by the above argument $f_{n} \in \overline{F(K)}$ for each $n$. Finally, since the sequence $\left\{f_{n}\right\}$ converges to $f$ in $L^{1}(E)$, $f \in \overline{F(K)}$.

1.4 Proposition. Let $\left\{I_{n}\right\}$ be an increasing sequence of closed ideals in $L^{1}(A)$. Then $\bigcup_{n} F\left(I_{n}\right)=F\left(\bigcup_{n} I_{n}\right)$.

Proof. If $f \in F\left(\cup_{n} I_{n}\right)$ define $f_{n}$ to be $f$ on $f^{-1}\left(I_{n}\right)$ and zero off it. Then the sequence $\left\{f_{n}\right\}$ converges to $f$ in $L^{1}(E)$, and $f_{n} \in F\left(I_{n}\right)$ for each $n$. Hence $f \in \widehat{\bigcup_{n} F\left(I_{n}\right)}$. Thus $F\left({\widetilde{\left.U_{n} I_{n}\right)}}_{F\left(\bigcup_{n} I_{n}\right.}\right) \subset \overline{\bigcup_{n} F\left(I_{n}\right)}$, by Proposition 1.3. For each $n, F\left(I_{n}\right) \subset F\left({\widetilde{U_{n} I_{n}}}_{1}\right)$ and hence, since the latter is closed, $\bar{U}_{n} F\left(I_{n}\right) \subset F\left({\widetilde{U_{n} I}}_{n}\right)$.

II. The partial Fourier transform. As a result of viewing $E$ measure theoretically as a product of $A$ and $G$ we can define a partial Fourier transform on $L^{1}(E)$. We first consider some preliminaries.

2.1 Definition. Let $X$ be a topological space and $E$ a Banach space.

(1) Let $B(X, E)$ denote the space of all bounded functions from $X$ to $E$ with the sup-norm $\|f\|_{\infty}=\sup \{\|f(x)\|: x \in X\}$. Under this norm, $B(X, E)$ becomes a normed space.

(2) Let $C(X, E)$ denote the subspace of $B(X, E)$ consisting of continuous functions.

(3) Let $C_{0}(X, E)$ denote the subspace of $C(X, E)$ consisting of functions which vanish at infinity.

2.2 Proposition. (1) $B(X, E)$ is a Banach space. 
(2) $C(X, E)$ and $C_{0}(X, E)$ are each closed subspaces of $B(X, E)$ and bence are Banach spaces.

The proofs are omitted.

Let $\hat{A}$ denote the dual of $A$. Then we shall be especially concerned with the space $C_{0}\left(\hat{A}, L^{1}(G)\right)$.

2.3 Definition. Let $f \in L^{1}(E)$. Define a map $\Phi: L^{1}(E) \rightarrow C_{0}\left(\hat{A}, L^{1}(G)\right)$ by

$$
\Phi f(a)(x)=\hat{f}(a)(x)=\int_{A} f(t, x) \bar{a}(t) d t \quad \text { for } a \in \hat{A} .
$$

The map $\Phi$ is called the partial Fourier transform of $L^{1}(E)$.

We must show that $\Phi$ has certain convenient and expected properties.

2.4 Proposition. (1) $\Phi$ is a norm-decreasing linear transformation from $L^{1}(E)$ into $C_{0}\left(\hat{A}, L^{1}(G)\right)$.

(2) $\Phi$ is one-to-one.

Proof of (1). Let $f \in L^{1}(E)$ and $a \in \hat{A}$. Then

$$
\|\hat{f}(a)\|=\int_{G}\left|\int_{A} f(t, x) \bar{a}(t) d t\right| d x \leq \int_{G} \int_{A}|f(t, x)| d t d x=\|f\|_{1} .
$$

Hence $\Phi$ is norm-decreasing.

Next let $D$ be the subspace of $L^{1}(E)$ spanned by all functions of the form $b \otimes f$, where $b \in L^{1}(A), f \in L^{1}(G)$, and $b \otimes f(t, x)=b(t) f(x)$. Then $(b \otimes f) \hat{\gamma}(a)=$ $\hat{b}(a) f$, where $\hat{b}$ is the usual Fourier transform of $b$ and $a \in A$. Hence $(b \otimes f)^{\wedge} \in C_{0}\left(A, L^{1}(G)\right)$ since $\hat{b} \in C_{0}(A)$, the space of continuous complexvalued functions on $A$ which vanish at infinity. Thus $\Phi(D) \subset C_{0}\left(\hat{A}, L^{1}(G)\right)$. Finally since $D$ is dense in $L^{1}(E)$, the first part follows.

Proof of (2). Since $\hat{A}$ is separable choose a countable dense subset $D$ of $\hat{A}$. For each $a \in \hat{A}$ let $I_{a}=\left\{b \in \dot{L}^{1}(A): \hat{b}(a)=0\right\}$. Let $K_{a}=F\left(I_{a}\right)$. Now suppose $f \in$ kernel $\Phi$. Then $f \in \bigcap_{a \in A} K_{a} \subset \bigcap_{d \in D} K_{d}$. For each $d \in D$ let $G_{d}=$ $\left\{x \in G: f(x) \notin I_{d}\right\}$. Let $G_{0}=\bigcup_{d \in D} G_{d}$. Then $G_{0}$ has measure zero and hence $f(x) \in \bigcap_{d \in D} I_{d}$ for almost all $x \in G$. Since $\bar{D}=A, \bigcap_{d \in D} I_{d}=\bigcap_{a \in A} I_{a}=0$. Hence $f=0$ a.e.

2.5 Proposition. The image of $L^{1}(E)$ under $\Phi$ is dense in $C_{0}\left(\hat{A}, L^{1}(G)\right)$.

Proof. By $\left[2\right.$, p. 375] any function in $C_{0}\left(\hat{A}, L^{1}(G)\right)$ with compact support can be approximated uniformly by finite sums of functions of the form $\Sigma k_{i} \otimes f_{i}$ where $k_{i} \in C_{0}(\hat{A})$ and has compact support, and $f_{i} \in L^{1}(G)$. Since the image under the Fourier transform of $L^{1}(A), L^{1}(A)^{\wedge}$, is dense in $C_{0}(\hat{A})$, any function in $C_{0}\left(\hat{A}, L^{1}(G)\right)$ with compact support can be approximated by functions of the form $\Sigma \hat{b}_{i} \otimes f_{i}$, where $b_{i} \in L^{1}(A)$, and $f_{i} \in L^{1}(G)$. But $\Sigma \hat{b}_{i} \otimes f_{i}=\left(\Sigma b_{i} \times f_{i}\right)^{\wedge}$ and 
hence the image of $L^{1}(E)$ is dense in the family of functions in $C_{0}\left(\hat{A}, L^{1}(G)\right)$ with compact support. Therefore we need only show that the family of functions in $C_{0}\left(\hat{A}, L^{1}(G)\right)$ with compact support is dense in all of $C_{0}\left(\hat{A}, L^{1}(G)\right)$.

Since $\hat{A}$ is second countable we can produce an increasing sequence $\left\{W_{n}\right\}$ of open subsets whose union is $\hat{A}$, whose closures are compact and such that $\bar{W}_{n} \subset W_{n+1}$ for all $n$. For each $n$ we can choose a function $k_{n} \in C_{0}(\hat{A})$ such that $k_{n}$ has value 1 on $W_{n}, 0$ off $W_{n+1}$, and such that the range of $k_{n}$ lies in the closed interval $[0,1]$. Now let $f \in C_{0}\left(\hat{A}, L^{1}(G)\right)$. Then it is a simple matter to verify that the sequence $\left\{k_{n} f\right\}$ converges to $f$ in $C_{0}\left(\hat{A}, L^{1}(G)\right)$ and that each member of the sequence has compact support.

III. Maximal ideals of $L^{1}(E)$. We now characterize the maximal closed oneand two-sided ideals of $L^{1}(E)$. Recall that $\sigma$ is the 2-cocycle which determines the extension $E$ of $G$ by $A$. Now for any $a \in \hat{A}$, the composition of $a$ by $\sigma$, $a(\sigma)$, determines a Borel function from $G \times G$ into $T$, the circle group, and $a(\sigma)$ is also a 2-cocycle.

3.1 Definition. Let $\tau: G \times G \rightarrow T$ be any Borel 2-cocycle. Then define $L^{1}(G, \tau)$ to be the Banach space $L^{1}(G)$ together with a multiplication (T) defined by

$$
f(7) g(x)=\int_{G} \tau\left(x y, y^{-1}\right)^{-1} f(x y) g\left(y^{-1}\right) d y
$$

and a mapping of $L^{1}(G)$ onto itself defined by

$$
f^{*}(x)=\tau\left(x, x^{-1}\right) \tilde{f}(x)
$$

where $\sim$ is the usual involution on the group algebra $L^{1}(G)$.

3.2 Proposition. $L^{1}(G, \tau)$ is a Banach algebra with involution *.

The proof is simply a matter of verification and is left to the reader. As a result of the above, for each $a \in \hat{A}$ we have a Banach algebra, $L^{1}(G, a(\sigma))$.

3.3 Definition. For each $a \in \hat{A}$ define a mapping $\mu_{a}: L^{1}(E) \rightarrow L^{1}(G, a(\sigma))$ by $\mu_{a}(f)=\Phi(f)(a)=\hat{f}(a)$.

3.4 Proposition. For each $a \in \hat{A}, \mu_{a}$ is a norm decreasing Banach algebra *-bomomorphism of $L^{1}(E)$ onto $L^{1}(G, a(\sigma))$, with kernel $K_{a}=F\left(I_{a}\right)$.

Proof. It is easily seen that $\mu_{a}$ is linear and norm decreasing. For $f$ and $g$ in $L^{1}(E)$,

$$
\mu_{a}(f * g)(x)=\int_{A} \int_{G} \int_{A} f(s t \sigma(x, y), x y) g\left(t^{-1} \sigma\left(y, y^{-1}\right)^{-1}, y^{-1}\right) \bar{a}(s) d t d y d s .
$$

By a repeated application of Fubini's theorem and several translations of variables and the fact that $\bar{a}(\sigma)\left(x y, y^{-1}\right) \bar{a}(\sigma)(x, y)=\bar{a}(\sigma)\left(y, y^{-1}\right)$, we eventually obtain that 
$\mu_{a}(f * g)=\mu_{a}(f)(a(\sigma)) \mu_{a}(g)$. We omit the details. Hence $\mu_{a}$ is a homomorphism. Next observe that $\mu_{a}$ is onto. For let $f \in L^{1}(G)$. Then choose an $b \in L^{1}(A)$ such that $\hat{b}(a)=1$. Then $\mu_{a}(b \otimes f)=\hat{b}(a) f=f$.

Next we show that $\mu_{a}$ preserves involution. Let $\lambda$ be the Haar measure of $A$ and $\nu$ the Haar measure of $G$. Then $\lambda \times \nu$ is the Haar measure of $E=A \times G$. Let $\delta$ be the modular function associated with $\nu$ and $\Delta$ the modular function associated with $\lambda \times \nu$. We observe that $\Delta(t, x)=\delta(x)$ for all $(t, x) \in E$. Let $R$ and $X$ be Borel subsets of $A$ and $G$ respectively, each of finite measure. Let $g \in G$. Then

$$
\begin{aligned}
& \lambda \times \nu((R \times X)(1, g))=\Delta(1, g) \lambda(R) \nu(G) \\
& \quad=\lambda \times \nu(\{(r \sigma(x, g), x g): r \in R \text { and } x \in X\})=\mu(R) \nu(X g)=\mu(R) \nu(X) \delta(g) .
\end{aligned}
$$

Hence $\Delta(1, g)=\delta(g)$ for all $g \in G$. By a similar argument it is seen that $\Delta(t, 1)=1$ for all $t \in A$. Then for $(t, x) \in E, \Delta(t, x)=\Delta((t, 1)(1, x))=$ $\Delta(t, 1) \Delta(1, x)=\delta(x)$. With this preliminary out of the way, the rest is simply a matter of computation.

Finally, $\mu_{a}(f)=0$ iff $\hat{f}(a)=0$ iff $f_{x} \in I_{a}$ for almost all $x \in G$ (where $\left.f_{x}(t)=f(t, x)\right)$. Hence $\mu_{a}(f)=0$ iff $f \in K_{a}=F\left(I_{a}\right)$.

We now proceed to state and prove our principle theorem.

3.5 Theorem. (1) For any $a \in \hat{A}$ and any maximal closed left ideal $M_{a}$ in $L^{1}(G, a(\sigma)), M=\mu_{a}^{-1}\left(M_{a}\right)$ is a maximal closed left ideal in $L^{1}(E)$. Furthermore, $M$ is regular iff $M_{a}$ is.

(2) Let $\mathbb{M}_{a}$ be the family of all maximal closed left ideals in $L^{1}(E)$ obtained as inverse images under $\mu_{a}$ of maximal closed left ideals in $L^{1}(G, a(\sigma))$. Then for any maximal closed left ideal $M$ in $L^{1}(E), M \in \mathbb{M}_{a}$ iff $K_{a} \subset M$.

(3) For any maximal closed left ideal $M$ in $L^{1}(E)$ there exists a unique $a \in \hat{A}$ and a unique maximal closed left ideal $M_{a}$ in $L^{1}(G, a(\sigma))$ such that $M=\mu_{a}^{-1}\left(M_{a}\right)$.

(4) The above three statements remain valid if the word 'left' is replaced everywhere by the word 'right', or by the word 'two-sided'.

Proof. We shall prove the theorem for left ideals. The proofs for right or two-sided ideals are identical.

First note that $\mu_{a}$ induces a topological as well as an algebraic isomorphism of $L^{1}(E) / K_{a}$ and $L^{1}(G, a(\sigma))$. From this it follows that there is a one-to-one correspondence between maximal closed left ideals in $L^{1}(G, a(\sigma))$ and maximal closed left ideals in $L^{1}(E)$ containing $K_{a}$. This establishes (1) and (2).

The proof of (3) is nontrivial and will take up the rest of this section. First note that for at most one $a \in \hat{A}$ can $M$ be the inverse image under $\mu_{a}$ of a subset 
of $L^{1}(G, a(\sigma))$. For suppose $M=\mu_{a}^{-1}\left(M_{a}\right)=\mu_{b}^{-1}\left(M_{b}\right)$ where $a \neq b$. Then $K_{b} \subset M$, and hence $\mu_{a}^{-1}\left(\mu_{a}\left(K_{b}\right)\right) \subset M$. Choose $b \in L^{1}(A)$ such that $\hat{b}(a)=1$, and $\hat{b}(b)=0$. Let $f \in L^{1}(G)$. Then $b \otimes f \in L^{1}(E)$ and $\mu_{b}(b \otimes f)=\hat{b}(b) f=0$. Hence $b \otimes f \in K_{b}$. But $\mu_{a}(b \otimes f)=\hat{b}(a) f=f$. Hence $\mu_{a}$ maps $K_{b}$ onto $L^{1}(G, a(\sigma))$, and thus $\mu_{a}{ }^{-1}\left(\mu_{a}\left(K_{b}\right)\right)=L^{1}(E)$-contradicting the maximality of $M$. This establishes the uniqueness. Thus the proof of Theorem 3.5 rests on the following:

Each maximal closed left ideal in $L^{1}(E)$ contains a $K_{a}$ for some $a \in \hat{A}$.

The rest of the section is devoted to establishing this result.

We have seen that any $f \in L^{1}(E)$ can be viewed as a function from $G$ to $L^{1}(A)$. This enables us to define a continuous multiplication of $L^{1}(A)$ by $L^{1}(E)$ into $L^{1}(E)$ as follows: For $f: G \rightarrow L^{1}(A)$ and $b \in L^{1}(A)$ define $b \cdot f(x)=$ $b *(f(x))$, where $*$ is the usual convolution in $L^{1}(A)$. Then we prove

Lemma 1. For f, $g \in L^{1}(E)$ and $b \in L^{1}(A)$,

(1) $b \cdot(f * g)=(b \cdot f) * g=f *(b \cdot g)$, and

(2) if $M$ is a closed ideal in $L^{1}(E)$ (one or two-sided) and $f \in M$ then $b \cdot f \in M$.

Proof of (1). Let $a \in \hat{A}$. Then taking partial Fourier transforms

$$
\begin{aligned}
(b \cdot(f * g) \hat{)}(a) & =\hat{b}(a)(\hat{f}(a)(a(\sigma)) \hat{g}(a))=(\hat{b}(a) \hat{f}(a))(\overparen{a(\sigma)}) \hat{g}(a) \\
& =(b \cdot f)^{\wedge}(a)(a(\sigma)) \hat{g}(a)=((b \cdot f) * g)^{\wedge}(a) .
\end{aligned}
$$

Hence since the partial Fourier transform is one-to-one we obtain that $b \cdot(f * g)=$ $(b \cdot f) * g$. The second part of the equality is similarly obtained.

Proof of (2). Let $\left\{U_{n}\right\}$ be an approximate identity for $L^{1}(E)$. Suppose that $M$ is a closed left ideal. Let $f \in M$. Then $\left(b \cdot U_{n}\right) * f \in M$. Hence by (1), $b \cdot\left\{U_{n} * f\right) \in M$. But $\left\{b \cdot\left(U_{n} * f\right)\right\}$ converges to $b \cdot f$. Since $M$ is closed, $b \cdot f \in M$. The proof for right ideals works similarly, applying the second part of the equality in (1).

Lemma 2. For $S \subset \hat{A}$ define $K_{S}=\left\{f \in L^{1}(E): \hat{f}(a)=0\right.$ for $\left.a \in S\right\}$. Then the following bold:

(1) $K_{S}=\bigcap_{a \in S} K_{a}$, and bence $K_{S}$ is a closed two-sided ideal in $L^{1}(E)$.

(2) Let $I$ be any closed left ideal in $L^{1}(E)$. Then the following bold:

(a) If $K_{U} \subset I$ for every neighborbood $U$ of infinity then $I=L^{1}(E)$.

(b) Let $a \in \hat{A}$. If $K_{U} \subset I$ for every neighborbood $U$ of a, then $K_{a} \subset I$.

Proof. The proof of $(1)$ is obvious. Let $\left\{U_{n}\right\}$ be a countable base for the neighborhood system of infinity in $A$. Then by hypothesis, $K_{U_{n}}=F\left(I_{U_{n}}\right) \subset I$ for each $n$. Hence by Proposition 1.4, ${\overline{U_{n} F\left(I_{U_{n}}\right.}})=F\left(\overline{U_{n} I_{n}}\right)=F\left(L^{1}(A)\right)=$ 
$L^{1}(E) \subset I$. The second equality follows from $[17, \mathrm{p} .162]$. Thus $(2(\mathrm{a}))$ follows. The proof of $(2(b))$ is similar.

We are now prepared to complete the proof of Theorem 3.5. Let $M$ be a maximal closed left ideal in $L^{1}(E)$. Since $M$ is proper there exists, by Lemma 2 above, a neighborhood $U$ of infinity such that $K_{U} \not \subset M$. We suppose that $K_{a} \not \subset M$ for each $a \in \hat{A}$ and arrive at a contradiction, namely that $K_{U} \subset M$. Let $g \in K_{U}$. Let $C$ be the complement of $U$ in $A$. Then $C$ is compact, and the support of $\hat{g}$ is contained in $C$. The next part of this argument is inspired by $[17$, p. 134]. Consider any $a \in \hat{A}$. Since $K_{a} \not \subset M$, there exists, by Lemma 2 , a neighborhood $U_{a}$ of $a$ such that $K_{U_{a}} \not \subset . M$. For each $a \in \hat{A}$ choose a neighborhood $0_{a}$ of $a$ and a function $b_{a} \in L^{1}(A)$ such that $\overline{0}_{a} \subset U_{a}$ and $\hat{b}_{a}$ has value 1 on $\overline{0}_{a}$ and 0 off $U_{a}$. Since $C$ is compact cover it with a finite number of $0_{a}$ 's say, $0_{1}, 0_{2}$, $\cdots, 0_{n}$. Define a function $\phi$ on $A$ by

$$
\phi=\left(1-\left(1-\hat{b}_{1}\right)\left(1-\hat{b}_{2}\right) \cdots\left(1-\hat{b}_{n}\right)\right) \hat{g} .
$$

Then it is easily verified that $\phi=\hat{g}$. Finally we argue that $\hat{g}=\phi \in \hat{M}=\Phi(M)$, and hence $g \in M$. Applying Lemma 1 we see that it suffices to argue that $b_{i} \cdot g \in M$ for each $i$. Fix any $i$. Since $K_{U_{i}} \not \subset M$ and $M$ is maximal, $K_{U_{i}}+M$ is dense in $L^{1}(E)$ and hence there exist sequences $\left\{f_{n}\right\}$ in $M$ and $\left\{k_{n}\right\}$ in $K_{U_{i}}$ such that $\left\{f_{n}+k_{n}\right\}$ converges to $g$. Then $\left\{b_{i} \cdot f_{n}+b_{i} \cdot k_{n}\right\}$ converges to $b_{i} \cdot g$. Now $\hat{k}_{n}$ is 0 on $U_{i}$ and $\hat{b}_{i}$ is 0 off $U_{i}$. Hence $b_{i} \cdot k_{n}=0$ for all $n$. Hence $\left\{b_{i} \cdot f_{n}\right\}$ converges to $b_{i} \cdot g$. Hence by Lemma $1, b_{i} \cdot g \in M$. Thus we can conclude that $g \in M$. Hence $K_{U} \subset M$, a contradiction. We conclude the theorem.

IV. Applications. Several applications of Theorem 3.5 are immediate. They involve relating 'local' and 'global' properties of the group extension. $a \in \hat{A}$.

4.1 Theorem. $L^{1}(E)$ is semisimple if $L^{1}(G, a(\sigma))$ is semisimple for each

4.2 Theorem. Eacb maximal closed ideal in $L^{1}(E)$ is regular iff the corresponding property is true for $L^{1}(G, a(\sigma))$ for each $a \in \hat{A}$.

The proofs of Theorems 4.1 and 4.2 are now obvious, based on Theorem 3.5.

4.3 Corollary. If $G$ is discrete then each maximal closed ideal in $L^{1}(E)$ is regular.

Proof. Since $G$ is discrete $L^{1}(G, a(\sigma))$ has an identity for each $a \in \hat{A}$. Hence each maximal ideal in $L^{1}(G, a(\sigma))$ is regular. Now apply Theorem 4.2.

We now prepare the way for a Bochner type theorem for continuous positive definite functions on $E$. But first we must establish a bound for the spectral norm on $L^{1}(E)$ (for a definition see $\left[12\right.$, p. 75]). For $\phi \in C_{0}\left(\hat{A}, L^{1}(G)\right)$ we define 
$\|\phi\|_{\infty}=\sup \{\|\phi(a)\|: a \in \hat{A}\}$. Then the following could be taken as a corollary to Theorem 3.5.

4.4 Theorem. For any $f \in L^{1}(E),\|f\|_{s \mathrm{p}} \leq\|\hat{f}\|_{\infty}$.

Proof. Let $f \in L^{1}(E)$, and suppose $\lambda \neq 0$ lies in the spectrum of $f$. Then by $[12, p .64]$ at least one of the following two statements must hold:

(1) There exists a regular maximal right ideal such that $(1 / \lambda) f$ modulo this ideal is a left identity.

(2) There exists a regular maximal left ideal such that $(1 / \lambda) f$ modulo this ideal is a right identity.

Suppose (1) holds. Let $I$ be such an ideal. Then $I$ is a maximal closed right ideal in $L^{1}(E)$ and hence by Theorem 3.5 there exists an $a \in \hat{A}$ such that $K_{a} \subset I$. Then $\mu_{a}(I)$ is a maximal closed regular ideal in $L^{1}(G, a(\sigma))$ modulo which $\mu_{a}((1 / \lambda) f)=(1 / \lambda) \hat{f}(a)$ is a left identity. Then by [12, p. 168], $\|(1 / \lambda) \hat{f}(a)\| \geq 1$. The same conclusion is reached if $(2)$ holds. Hence for any nonzero member $\lambda$ of the spectrum of $f$ there exists an $a \in \hat{A}$ such that $|\lambda| \leq\|\hat{f}(a)\|$. The conclusion follows.

4.5 Corollary. For any $f \in L^{1}(E), \lim _{n \rightarrow \infty}\|f\|^{1 / n} \leq\|\hat{f}\|_{\infty}$.

Proof. Apply Theorem 4.4 to $[12$, p. 75].

Let $X$ be a Borel space and $\mu$ a positive measure defined on the Borel subsets of $X$. Let $\mathcal{C}(X, \mu)$ denote the family of $\mu$-equivalence classes of complex-valued Borel functions on $X$. If $X$ and $Y$ are two Borel spaces with Borel measures $\mu$ and $\nu$ respectively and if $f$ is a complex-valued Borel function on $X \times Y$ we shall let $f^{\prime}$ denote the function from $X$ to $\mathcal{C}(Y, \nu)$ defined by (within equivalence) $f^{\prime}(x)(y)=f(x, y)$.

4.6 Proposition. For eacb $f \in C\left(A, L^{\infty}(G)\right)$ there exists a Borel function $g \in L^{\infty}(A \times G)=L^{\infty}(E)$ such that $g^{\prime}(t)=f(t)$ for almost all $t \in A$.

Proof. First suppose that $f$ has compact support. Then as a function from $A$ to $L^{\infty}(G), f$ is integrable with respect to the Haar measure on $A$. Hence by [3, p. 198] a function $g$ exists such that $g^{\prime}=f$. It is clear that $g$ is essentially bounded and hence $g \in L^{\infty}(A \times G)$.

Now suppose that $f$ does not have compact support. Then choose a sequence $\left\{k_{n}\right\}$ of functions in $C_{0}(A)$ as was done in the proof of Proposition 2.5. Then since $k_{n} f$ has compact support for each $n$, there exists a complexvalued Borel function $g_{n}$ on $A \times G=E$ such that $g_{n}^{\prime}=k_{n} f$ a.e. on $A$. Now define a sequence $\left\{b_{n}\right\}$ of functions on $A \times G$ inductively as follows: $b_{1}=g_{1}$, and for all $n, b_{n+1}(t, x)=b_{n}(t, x)$ for $t \in W_{n}$ and $b_{n+1}(t, x)=g_{n+1}(t, x)$, 
otherwise. Then $\left\{b_{n}\right\}$ converges a.e. to a Borel function $g$ on $A \times G=E$ and $g^{\prime}=f$ for almost all $t \in A$. Again it is clear that $g$ is essentially bounded and hence $g \in L^{\infty}(A \times G)=L^{\infty}(E)$.

Let $f$ be any bounded complex-valued uniformly continuous function on $E$. Since $E$ can be viewed as the product $A \times G$ of two Borel spaces, $f$ determines a function $f^{\prime}$ from $A$ into $L^{\infty}(G)$.

4.7 Proposition. $f^{\prime}$ lies in $C\left(A, L^{\infty}(G)\right)$.

Proof. As a preliminary recall that in the extension $1 \rightarrow A \rightarrow E \rightarrow G \rightarrow 1$, $A$ is mapped into $E$ by $i$, where $i(a)=(a, 1)$, where 1 is the identity of $G$. Now consider any $\epsilon>0$. Then there exists a neighborhood $U^{\prime}$ of the identity of $E$ such that for any $w, z \in E$ if $w z^{-1} \in U^{\prime}$ then $|f(w)-f(z)|<\epsilon$. Let $U=$ $i^{-1}\left(U^{\prime} \cap i(A)\right)$. Then $U$ is a neighborhood of the identity in $A$. Let $s, t \in A$ such that $s t^{-1} \in U$. Then for any $x \in G,(s, x)(t, x)^{-1}=(s, x)\left(\left(t \sigma\left(x, x^{-1}\right)\right)^{-1}, x^{-1}\right)$ $=\left(s t^{-1}, 1\right)$. Hence for any $x \in G,(s, x)(t, x)^{-1} \in U^{\prime}$ and hence, $|f(s, x)-f(t, x)|<\epsilon$. We conclude that $\left\|f^{\prime}(s)-f^{\prime}(t)\right\|_{\infty} \leq \epsilon$. Hence $f^{\prime}$ is continuous.

It is well known that a continuous positive definite function on $E$ is uniformly continuous. For $t \in A$ and $a \in \hat{A}$ we shall use the standard notation to denote the continuous map from $A \times \hat{A}$ to $T$ by $(t, a)=a(t)$. We are now prepared to present the Bochner theorem.

4.8 Theorem. Let $\phi$ be a continuous positive definite complex-valued function on $E$. Then there exists a regular measure $m$ on $\hat{A}$ of finite variation with values in $L^{\infty}(G)$ such that

$$
\phi^{\prime}(t)=\int_{\hat{A}}(t, a) d m(a) \text { for all } t \in A .
$$

Proof. To as great an extent as possible we mimic the proof of the standard Bochner theorem as provided in [17, p. 19], but substituting the Fourier analysis we have developed. We refer freely to this proof.

The first several paragraphs of Rudin's proof carry over except that we use multiplicative notation and integrate over $E$. As in [17] we assume without loss of generality that $\|\phi\|_{\infty}=1$. We must take care in verifying (6) of $[17$, p. 20] to include the modular function of $E$ when taking the involution of $g$. Thus defining $T_{\phi}(f)=\int_{E} f(x) \phi(x) d x$ for $f \in L^{1}(E)$ we have ((8) of [17, p. 20]) $\left|T_{\phi}(f)\right|^{2} \leq T_{\phi}(f * \widetilde{f})$ for all $f=L^{1}(E)$. Then as in [17, p. 21] putting $b=f * \widetilde{f}$ and $\dot{b}^{n}=b^{n-1} * b$ for all $n>1$ we conclude

$$
\left|T_{\phi}(f)\right|^{2} \leq T_{\phi}(b) \leq\left\{T_{\phi}\left(b^{2^{n}}\right)\right\}^{2^{-n}} \leq\left\|b^{2^{n}}\right\|^{2^{-n}} \text { for all } n,
$$

Hence by Corollary 4.5 we obtain $\left|T_{\phi}(f)\right|^{2} \leq\|\hat{b}\|_{\infty}$. By a simple computation we 
note that $\|\hat{b}(a)\| \leq\|\hat{f}(a)\|^{2}$ for all $a \in \hat{A}$ and hence $\left|T_{\phi}(f)\right| \leq\|\hat{f}\|_{\infty}$. Thus since $\Phi: L^{1}(E) \rightarrow C_{0}\left(\hat{A}, L^{1}(G)\right)$ is one-to-one and $\Phi\left(L^{1}(E)\right)$ is dense in $C_{0}\left(A, L^{1}(G)\right)$, $T_{\phi}$ can be regarded as a bounded linear functional on all $C_{0}\left(A, L^{1}(G)\right)$. Then by $[2$, p. 387] there exists a regular measure $m$ with finite variation $v$ defined on the Borel subsets of $\hat{A}$ and with values in the dual of $L^{1}(G), L^{\infty}(G)$, such that $T_{\phi}(b)=\int_{\hat{A}} b\left(a^{-1}\right) d m(a)$ for all $b \in C_{0}\left(\hat{A}, L^{1}(G)\right)$. Now consider any $f \in L^{1}(A)$ and $g \in L^{1}(G)$. Then $f \otimes g \in L^{1}(E)$ and

$$
T_{\phi}(f \otimes g)=\int_{\hat{A}}(f \otimes g)^{\wedge}\left(a^{-1}\right) d m(a)=\int_{\hat{A}} \hat{f}\left(a^{-1}\right) d(g \cdot m)(a),
$$

where $g \cdot m$ is a complex-valued measure on $\hat{A}$ defined by $g \cdot m(S)=m(S)(g)$ for $S$ a Borel subset of $\hat{A}$. Hence

$$
\begin{aligned}
T_{\phi}(f \otimes g) & =\int_{\hat{A}} \hat{f}\left(a^{-1}\right) d(g \cdot m)(a)=\int_{A} f(t) \int_{\hat{A}}(t, a) d(g \cdot m)(a) d t \\
& =\int_{A} f(t) g \cdot\left(\int_{\hat{A}}(t, a) d m(a)\right) d t .
\end{aligned}
$$

Here $\int_{\hat{A}}(t, a) d m(a)$ is an element of $L^{\infty}(G)$ and $g \cdot\left(\int_{\hat{A}}(t, a) d m(a)\right)$ is the image of $g$ under this linear functional. The above equations follow by repeated application of Theorem $(8(f))$ of $[3$, p. 324]. Now put

$$
p(t)=\int_{\hat{A}}(t, a) d m(a) \text { for all } t \in A \text {. }
$$

Then $p$ maps $A$ into $L^{\infty}(G)$ and $T_{\phi}(f \otimes g)=\int_{A} f(t)(g \cdot p(t)) d t$. Next, we concern ourselves with showing that $p \in C\left(A, L^{\infty}(G)\right)$. For any $t \in A,\|p(t)\|_{\infty}=$ $\left\|\int_{\hat{A}}(t, a) d m(a)\right\| \leq v(\hat{A})$, where $v$ is the total variation of $m$, and hence $p$ is bounded. Since $m$ is regular, $v$ is left regular [2, Proposition 19, p. 3.14]. Now consider any $\epsilon>0$. Then there exists a compact set $C \subset \hat{A}$ such that $v(\hat{A} \sim C)$ $<\epsilon / 4$. Also there exists a neighborhood $V$ of the identity in $A$ such that, for $s t^{-1} \in V$ and $a \in C,|a(s)-a(t)|<\epsilon / 2 v(C)$. Then for $s t^{-1} \in V$,

$$
\begin{aligned}
\|p(s)-p(t)\|_{\infty} & =\left\|\int_{\hat{A}}(a(s)-a(t)) d m(a)\right\| \\
& \leq \int_{\hat{A}}|a(s)-a(t)| d v(a) \\
& =\int_{C}|a(s)-a(t)| d v(a)+\int_{\hat{A} \sim_{C}}|a(s)-a(t)| d v(a) \\
& \leq \epsilon / 2+\epsilon / 2=\epsilon .
\end{aligned}
$$

Thus $p \in C\left(A, L^{\infty}(G)\right)$. Hence by Proposition 4.6 there exists a Borel function $q \in L^{\infty}(E)$ such that $q^{\prime}=p$ a.e. Then for $g \in L^{1}(G), g \cdot p(t)=\int_{G} g(x) q(t, x)$ for almost all $t \in A$. Hence for all $f \in L^{1}(A)$ and $g \in L^{1}(G)$, 


$$
T_{\phi}(f \otimes g)=\int_{A} f(t)(g \cdot p(t)) d t=\int_{A} \int_{G} f(t) g(x) q(t, x) d x d t=\int_{E} f \otimes g(z) q(z) d z
$$

Hence $T_{\phi}=T_{q}$ on functions of the form $f \otimes g$ for $f \in L^{1}(A)$ and $g \in L^{1}(G)$. Since these functions generate a dense subspace of $L^{1}(E), T_{\phi}=T_{q}$ on all $L^{1}(E)$. Hence $\phi=q$ a.e. Hence $\phi^{\prime}(t)=p(t)$ for almost all $t$. Thus by Proposition 4.6 and the fact that $p$ is continuous, $\phi^{\prime}=p$. Hence $\phi^{\prime}(t)=\int_{\hat{A}}(t, a) d m(a)$ for all $t \in A$. This completes the proof.

\section{BIBLIOGRAPHY}

1. L. Auslander and C. C. Moore, Unitary representations of solvable Lie groups, Mem. Amer. Math. Soc. No. 62 (1966). MR 34 \#7723.

2. N. Dinculeanu, Vector measures, Internat. Series of Monographs in Pure and Appl. Math., vol. 95, Pergamon Press, Oxford; VEB Deutscher Verlag der Wissenschaften, Berlin, 1967. MR 34 \#6011b.

3. N. Dunford and J. T. Schwartz, Linear operators. I: General theory, Pure and Appl. Math. vol. 7, Interscience, New York, 1958. MR 22 \#8302.

4. P. R. Halmos, Measure theory, Van Nostrand, Princeton, N. J., 1950. MR 11, 504.

5. A. Hausner, On generalized group algebras, Proc. Amer. Math. Soc. 10 (1959), 1-10. MR $21 \# 3777$.

6. G. P. Johnson, Spaces of functions with values in a Banach algebra, Trans. Amer. Math. Soc. 92 (1959), 411-429. MR 21 \#5910.

7. K. B. Laursen, Ideal structure in generalized group algebras, Pacific. J. Math. 30 (1969), 155-174. MR 40 \#1792.

8. H. Leptin, Verallgemeinerte $L^{1}-$ Algebren, Math. Ann. 159 (1965), 51-76. MR $39 \# 1909$.

9. - Verallgemeinerte $L^{1}$-Algebren und projektive Darstellungen lokal kompakter Gruppen. I, Invent. Math. 3 (1967), 257-281. MR 37 \#5328.

10. - Verallgemeinerte $L^{1}-$ Algebren und projektive Darstellungen lokal kompakter Gruppen. II, Invent. Math. 4 (1967), 68-86. MR 37 \#5328.

11. - Darstellungen verallgemeinerter $L^{1}-$ Algebren, Invent. Math. 5 (1968), 192-215. MR 38 \#5022.

12. L. Loomis, An introduction to abstract harmonic analysis, Van Nostrand, Princeton, N. J., $1953 . \quad$ MR 14, 883.

13. G. W. Mackey, Borel structure in groups and their duals, Trans. Amer. Math. Soc. 85 (1957), 134-165. MR 19, 752.

14. - Les ensembles boréliens et les extensions des groupes, J. Math. Pures Appl. (9) 36 (1957), 171-178. MR 19, 752.

15. - The theory of group representations, Lecture Notes, Dept. of Math., Univ. of Chicago, Chicago, Ill., 1955. MR 19, 117.

16. C. C. Moore, Extensions and low dimensional cohomology theory of locally compact groups. I, Trans. Amer. Math. Soc. 113 (1964), 40-63. MR 30 \#2106.

17. W. Rudin, Fourier analysis on groups, Interscience Tracts in Pure and Appl. Math., no. 12, Interscience, New York, 1962. MR 27 \#2808.

DEPARTMENT OF MATHEMATICS, ILLINOIS STATE UNIVERSITY, NORMAL, ILLINOIS 